\title{
A Conversation Analysis Approach to Attributable Silence in Moroccan Conversation
}

\author{
Samira Elouakili (Corresponding author) \\ Ibn Tofail University, School of Humanities, Kenitra, Morocco \\ E-mail: samielouakili@yahoo.fr
}

Received: April 22, 2017 Accepted: May 12, 2017 Published: May 31, 2017

doi:10.5296/ire.v5i2.11369 URL: http://dx.doi.org/10.5296/ire.v5i2.11369

\begin{abstract}
This paper is an adaptation of one section in the theoretical part of a MA thesis on 'The conversational role of silence in Moroccan Arabic' obtained in 1990, and aims to account for attributable silence (Schegloff \& Sacks, 1973) within the conversation analysis approach based on the turn-taking model advanced by Sacks, Schegloff, and Gefferson (1974). Attributable silence occurs when a speaker is selected to speak upon the completion of an utterance that solicits a particular response but chooses, for one reason or another, to remain silent. Systematic and comprehensive as the model has often been claimed to be, it does not deal with this category of silence, which is highly significant to conversation partners in daily interaction. Hence, we attempt to provide an account for it using some of the turn-taking mechanisms developed within the model itself. This silence is characterized as a violation of the turn-taking rule involving the current speaker selects next technique, and the repair of the violation is provided through the suggestion of a rule stipulating that if a selected speaker fails to start a next turn, then the current speaker has the right to pursue a response until he obtains one; otherwise, the conversation may discontinue. Features that reveal the significance of this silence are also discussed-namely noticeability, attributability, accountability, and reportability. The examples used to discuss and illustrate these points are taken from the observational and experimental data collected for the thesis.
\end{abstract}

Keywords: accountability, attributable silence, current speaker selects next technique, repair, turn-taking system violation

\section{Introduction}

Silence is "as intrinsic to human existence as is speech" (Kenny, 2011, p. 12). It is not merely the absence of sound. In fact, it is laden with meanings. Suffice it to look at a culture's verbal heritage such as idiomatic expressions and proverbs to realise the myriad of meanings this absence of sound conveys. This variety may be attributed to different factors. One is the fact 
that silence is "highly culture-dependent"; another is it is not a unified phenomenon; there rather exist "varieties of silence" (Shrader-kniffki, 2007, p. 305). Shrader-Kniffki (2007) deals with it in relation to politeness in a cross-cultural contex-Spanish/Zapotec Mexican interactions. Levinson and Torreira (2015) consider the interaction between inter-turn silences and language processing and production. Other research reports that cultures and subcultures might differ in what the "unmarked value of a silence" between turns is and the way "they "reckon" the invisible, normative beat between one turn and the next" (Schegloff, 2015, p. 348). Stiver et al. (2009) claim that the reported differences are presumably due to the subjective perception of cross-cultural beat differences, and more precisely to outsiders' "sensitivity to subtle variation" (conclusion, para. 2), rather than to actual significant differences. They examine response latency patterns after polar questions in ordinary conversation in ten languages spoken in both traditional indigenous communities and major others, and find evidence that turn transfer is achieved in all these languages with minimal silence and overlap. Contrary to anthropological reports, they observe significant universal patterns of response latency.

In this paper, we examine attributable silence (AS) within the conversation analysis framework using Sacks et al.'s model (1974). One advantage of the latter is that it does not view silence as one phenomenon; it, instead, considers it as an interactional event the nature and significance of which is determined by its placement in the ongoing talk. Thus, it identifies different types of silence-pauses, gaps, and lapses; however, it does not handle AS. Hence, using mechanisms advanced within the model itself and data from recorded conversations, field notes, and an experiment on participants' reaction to and tolerance interval of AS, we attempt to extend the model so that it can account for this silence and the repair of the breach of the turn-taking machinery that it represents within the system itself rather than outside it, in line with one of Sacks et al.'s (1974) main principles in constructing the model. We first introduce some relevant basic components of the system. Then we provide our extension and finally expose major features of this category of silence.

\section{The Sequential Approach}

Sacks et al. (1974)'s model and conversation analysis in general deal with silence within the context of daily interaction, which, as Levinson \& Torreira (2015) state, is "the prime ecological niche for language, the context in which language is learned [.......] in which the cultural forms of language have evolved, and where the bulk of language usage happens" (introduction, para 1). Their model, which is based on conversations audio-taped in natural contexts, represents a systematic and comprehensive account of conversational events and has so far proved to work across different languages and contexts. Departures from interactional patterns that are common in industrialized Western societies involve what might be referred to as "differences in the values of variables" (Schegloff, 2015, p. 348), not in the variables or the turn-taking practices themselves nor their organisation. In fact, Stiver et al.'s (2009) study and Gardner \& Mushin's (2015) investigation of expanded transition places in Aboriginal Australian conversation in the Garrwa language, among others, confirm that Sacks et al.'s (1974) fundamental turn-taking mechanisms are universal and that the across-language differences are only quantitative. 
The sequential model deals, for instance, with how turns are exchanged, how they are constructed, how speakers are interrupted and how this interruption is resolved, and how different types of silence obtain. They assume that conversation operates on a turn by turn basis. Participants generally alternate turns and finely coordinate transition with no or minimal gaps and overlaps. Briefly, the model consists of two main components-the constructional and the turn allocation components.

The first component concerns the units out of which participants construct their turns, and these, according to Sacks et al. (1974), are syntactic units-sentences, clauses, phrases, and words, though they acknowledge the importance of other components in this context: "Some understanding of sound production (i.e., phonology, intonation, etc.) is also very important to turn-taking organization" (p. 721). Around the ends of these units transfer may occur. These loci, which they call transition relevance places (TRPs), are potential for not at any TRP would a participant take his turn. By having a turn, a speaker is "initially" entitled to one such unit, which implies he may employ more than one before his co-participant intervenes. He may also expand his unit types, especially the sentential one. The basic characteristic of these units is that their end is projectable: Listeners can project the potential completion of a turn through their analysis of the unit as it unfolds in time. They can undertake this analysis by virtue of their knowledge of the language.

Turn projection has been criticized by some scholars such as Heldner \& Edlund (2010), but Levinson \& Torreira (2015) provide evidence that recipients do predict the end of turns as the speaker's utterances unfold. Reviewing extensive experimental research conducted over decades on language production, they find that latencies involved in language production average around $0.6 \mathrm{sec}$ whereas between-turn gaps tend to average around $0.2 \mathrm{sec}$. This as well as turn-completion by the other imply that listeners encode their response as their partners' utterances develop in time, which allows them to predict their ends and thus minimize between-turn gaps (Levinson \& Torreira, 2015, section 2, para. 13).

The conception of turns in structural terms has also been criticized on the grounds that turns do not necessarily consist of syntactic units; some authors claim that intonation plays a major role in delineating turns (Power \& Martello, 1986); others point to the crucial importance of message completion (Chafe, 1985). In their review of the literature on the issue, Levinson and Torreira 2015) conclude that turns may consist of interjections, words, phrases or clauses as well as intonational units and that "turn ends typically co-occur with points of both syntactic and prosodic completion" (see also Shegloff, 2007). Hence, we adopt Shegloff's (1972) definition: “A turn may contain anything from a single 'mm' (or less) to a string of complex sentences" (p. 376). Repair devices such as the ones uttered by current speakers like / $\mathrm{Pa} /$ (what?) in H4 in example (1) and L2 in (5) below are also considered turns. They are used to pursue a relevant response, and clearly suggest that the prior utterances by the current speaker were complete and that their recipients' responses should have appeared before the former resumed his talk.

The second component concerns how turn allocation operates. Participants have at their disposal a set of rules that help them coordinate turn transfer in such a way as to minimize 
gaps and overlaps. They stipulate that either speakers select themselves for next speakership on the occurrence of a TRP or are selected by a current speaker through different repair devices such as tags, repair initiators such as 'what?' and 'who?', and the first parts of adjacency pairs like request/granting or declining and summons/answer pairs (see below). The set of rules, the first of which, I (a), is relevant to us, is as follows:

I. For any turn, at the initial transition-relevance place of an initial turn-constructional unit:

(a) - If the turn so far is so constructed as to involve the use of a 'current speaker selects next' technique, then the party so selected has the right, and is obliged to take next turn to speak, and no others have such rights or obligations, and transfer occurs at that place.

(b) - If the turn so far is so constructed as not to involve the use of 'a current speaker selects next' technique, then self-selection for next speakership may, but need not, be instituted, first starter acquires rights to a turn, and transfer occurs at that place.

(c) - If the turn so far is so constructed as not to involve the use of 'a current speaker selects next' technique, then current speaker may but need not continue, unless another self selects.

II. If at the initial transition relevance place of an initial turn constructional unit neither I (a) nor I (b) has operated, and, following the provision of I (c), the current speaker has continued, then the rule set (a) - (c) reapplies at the next transition relevance place, and recursively at each next transition relevance place, until transfer is effected (p. 704).

As shown in the formulation of the rules, participants, as mentioned above, coordinate turn transfer around TRPs. If so is the case, Sacks et al. (1974) argue, then gaps and overlaps are predicted to obtain not at any point in a single turn, but between different turns and constructional units, and given participants' orientation to these rules and the predictability of the end of turn construction units, gaps tend to be slight. This slightness is accounted for within the system itself. Gaps, as pointed out earlier, are generally slight because of listeners' ability to analyze the speaker's turn as it develops and thereby to project its completion point. Besides, given the presence of rule 1 (c), a listener interested in talking would not allow much time, if any, to elapse, after a current speaker reaches a TRP; otherwise, the latter may take up his turn under rule 1 (c). Another way in which the rules minimize silence is the current speaker's resumption of his talk under rule 1 (c) after rule 1 (b) has been passed.

Using the turn constructional component and the turn allocation one, and taking into consideration the surrounding talk, the position silence occupies within conversation, and its length, Sacks et al. (1974) distinguish between gaps, pauses, and lapses. A lapse is an extended silence that emerges when the current speaker-selects next technique is not employed and at least one round of possible self-selection by others and continuation by current speaker may develop during which none of these options is used. Accordingly, the 
more rounds are passed the more extended the silence is.

A pause is generally understood to refer to a moment of silence bounded by talk of the same speaker whether it is at a TRP or not: "If a developing silence occurs at a transition-place, and is thus a (potential) gap, it may be ended by talk of the same party who was talking before it; so the 'gap' is transformed into a 'pause' (being now intra-turn)" (Sacks et, 1974, footnote, p. 715). However, a silence before a selected party starts his turn is also referred to as a pause: "A silence after a turn in which a next has been selected will be heard not as a lapse's possible beginning, not as a gap, but as a pause before the selected next speaker's turn-beginning" (p. 725). This we find a little confusing. Its classification as a pause implies that the selected party stops talking and then resumes his talk, which is not the case for this silence is the outcome of the operation of rule 1 (a). The label it is assigned then equates it with the intra-turn pause which obtains under the operation of rule 1 (c). A gap, as the former quote implies, is used to refer to an instance of silence at a TRP, or one bounded by talk of different speakers. Here lies another terminological problem for what is considered as a pause under the operation of rule 1 (a) is also bounded by talk of different speakers. Despite this terminological confusion, the sequential model, unlike some previous attempts within the psycholinguistic and the signalling approaches, identifies different types of silence in terms of rules and their ordered operation. In addition, it views silence as a dynamic phenomenon capable of transformation, and, like the turn, as the outcome of the collaboration of both the speaker and the hearer (Shegloff, 1982, p. 73).

Though the fundamentals of the turn-taking system such as participants' orientation to taking turns and the systematic speaker change are generally agreed on, the slightness of between-turn gaps has not been found to characterize all inter-turn transitions (see, for example, Kendrick, 2015 and Kendrick \& Torreira, 2015). Mushin \& Gardner (2009), Gardner (2010), and Gardner \& Mushin (2015) observe a significant variability in silence length in Garrwa talk and a high tolerance for lengthy between-turn silences in conversations involving elderly Aboriginal women. According to them, this tolerance could be a function of the close relation between the participants and the absence of any "particular orientation to the clock time" (Mushin and Gardner, 2009, p. 36). If so is the case, they claim, then this feature of conversational style might characterize any community that shares this kind of interpersonal relation (p. 37). More generally, they as well as Shegloff (2010) explain the occurrence of long silences in conversation in terms of the "non-focused" or "incipient" nature of the talk concerned, which is sporadic and characterized by lengthy silences. Such findings suggest that how a conversation develops depends on its placement in a given interaction context and "how an upcoming lapse in the operation of the basic features is attended to and dealt with by participants is sensitive to, and/or can accomplish, the placement of the conversation in its occasion" (Schegloff, 2010, pp. 46-47). Therefore, Shegloff (2010) states that we should not deconstruct the outcome of decades of work that has been accomplished in the field; we had better extend it so that it can account for features of incipient talk (p.47).

In fact, Sacks et al.'s model (1974) has generated a wealth of research on both the structural and temporal aspects of conversation. However, it does not provide for AS though, as stated 
earlier, it is highly significant in every day conversation. It does not talk about the sequential consequences of a selected speaker's opting not to honor rule I (a). We do not know, for instance, whether the current speaker simply exits from the conversation, pursues a response, or shifts to another topic. Nonetheless, the model and the conversation analysis approach in general provide mechanisms to identify and characterize this category of silence such as the current speaker selects next technique, one very important and recurrent example of which is, as mentioned previously, adjacency pairs. Before we examine these and their inherent relation to AS and expand the model, we introduce the reader to the different thesis data sources, from which the examples below are taken.

\section{Methodology}

One experimental and three observational methods were employed. Given that the significance of silence is largely determined by the sequential context it occurs in, we tape-recorded conversations in natural contexts. The participants were our female friends and relatives of both genders. Most of them were informed about the recording after it had been completed, at which none expressed their anger. During the recording, we tried to observe carefully and note down the interactants' non-verbal behavior such as gaze and facial expressions.

Tape recording, however, was time consuming and generated few instances of AS; we had, therefore, to use field notes though this method is often criticized as it depends on recollection. One may forget details that are of important relevance to one's study. To deal with this problem, we wrote down the relevant anecdotes as well as short conversational excerpts and the accompanying nonverbal behavior immediately upon their occurrence. The examples might be said not to be a perfect reflection of what actually happened, yet we can still confirm we were able to retain most of the significant features of the events. Furthermore, the method allowed us to collect examples in other natural contexts involving participants we had no relation with, which gave the data more variety.

To study the role of the nonverbal component, which is of major importance in silence interpretation, we video-recorded Moroccan series from three soap operas shown on Moroccan TV. The one from which two examples are discussed in this paper is entitled / lxil mrabətha/ (The horses' esteem (or honor) is in their barn). One might argue that the verbal and non-verbal acts are not very spontaneous or natural in such a context. They might, indeed, have been dramatized or based on stereotyped behaviors, yet the method enabled us to describe, with a fair degree of certainty, the nonverbal acts that accompanied different instances of silence and helped in their interpretation.

To examine participants' reaction to and tolerance interval of AS, we reacted by silence to requests for information and opinion made by fourteen female close friends and sisters aged between 18 and 38. The task was quite challenging for we had to maintain the same nonverbal behavior we adopted and the activity we were doing before the start of 'the game' so as not to allow other factors such as smiles and laughter to interfere with the interpretation of the silence. Once the attempt to continue to do so failed under the non-serious insulting prompts to provide a response, the 'game' stopped. Some of these sequences were recorded 
and others were noted down immediately the 'game' ended.

None of the methods above can be claimed to be perfect; nonetheless, their combination can generate some valid conclusions. Whenever possible, instances of AS were measured three times with a stop watch and averaged. The transcription is rather simplified featuring only the participants' utterances, some of their nonverbal acts when relevant to the analysis, and the silences measured in seconds. (See the appendix for the keys to the transcription symbols and the abbreviations for the data sources).

\section{Adjacency Pairs}

As mentioned previously, first parts of adjacency pairs are a basic mechanism of the current speaker selects next technique. They are paired utterances the component parts of which are produced by different speakers one after the other, and have a particular relatedness in that "given the recognizable production of a first pair part, on its first possible completion, its speaker should stop and a next speaker should start and produce a second pair part from the pair type of which the first is recognizably a member" (Schegloff \& Sacks, 1973, p. 296).

The adjacency feature has been criticized on the grounds that first pair parts are not always immediately followed by second pair parts (Owen, 1981; Levinson, 1983). There often emerges some talk between the two parts. However, this talk is usually understood as being "oriented to" the action performed in the first pair part and is scanned for its relevance to it (Owen, 1981, p. 104. See also Shegloff, 2007), as illustrated by the following example.

1. H1: samira nəddi 33əllaba djalək ?

Samira, can I have your Jellaba?

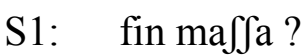

Where are you going ?

H2: $\quad$ Gand wahəd lbənt

To a girl's

S2: Jkun had lbənt?

Which girl?

H3: hadik lli Sanda tfanḍar ləћmar djali

The one who has my red training-suit

(silence)

H4: Pa?

what?

(silence)

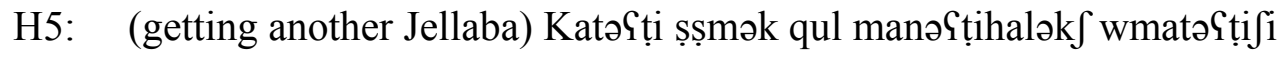

You're turning a deaf ear to me. Say I won't give it to you and don't

(NREE)

To account for cases like these as well as others where the parts come in more than two turns, Shegloff (2007) argues for the replacement of the adjacency feature present in the basic form cited above by conditional relevance in the following way: 
"First" and "second" do not refer merely to the order in which these turns happen to occur; they refer to design features of these turn types and sequential positions. The very feature of "first-ness" sets up the relevance of something else to follow; it projects the relevance of a "second." It is the occurrence of a first pair part that makes some types of second pair part relevant next; that relevance is conditioned by the FPP [first pair part]. If such a second pair part is produced next, it is heard as responsive to the first pair part which preceded. If such a second pair part is not produced next, its non-occurrence is as much an event as its occurrence would have been. It is, so to speak, noticeably, officially, consequentially, absent (p. 20). (The emphasis is ours).

Thus first parts of adjacency pairs impose expectations of fulfilment in the sense that they set up an expectation of a relevant response upon their completion. If the selected speaker opts for silence as a response to a first pair part, this absence of talk is "an event in its own right" (Shegloff, 2007, p. 21). Absence of talk, in this case, is noticeable for it is "officially absent" Shegloff (1972, pp. 388/389). Thus, first pair parts not only constrain powerfully what selected speakers should do but also how whatever they do, keeping silent being one option, should be interpreted by their co-participants (Shegloff, 2007, p. 21)

One piece of evidence for the 'noticeability' of AS is that Mushin \& Gardner (2015) observe that though Aboriginal people do tolerate periods of silence as long as $13 \mathrm{sec}$ and treat them as ordinary, a silence of just $1.5 \mathrm{sec}$ after the operation of rule 1 (a) indicates trouble (p. 34). In fact, AS is often referred to as meaningful silence. Levinson \& Torreita (2015), for instance, refer to a silence after a question as a "meaningful absence of speech" (section 2, para. 7).

A silence after a summons as in example (2) below is also noticeable and meaningful for the selector notices his failure to mobilize the attention of the recipient, upon which depends the occurrence of a further action-the one he was summoned for (Shegloff, 2007, p. 62). In such cases, the summoner signals this failure, as demonstrated by example (2). Upon receiving no answer to her summons in M1, M, H's mother, repeats it in M2 prefacing it with an attention-getting device in Moroccan Arabic /Ra:/ (Hey!) and expresses her concern about him in M3. / kbitti/ (literally, my liver) is an affectionate term of address.

2. M1 : ћməd?

Hmed ?

$(0.75 \mathrm{sec})$

M2 : Pa: ћməd!

Hey Hmed!

$\mathrm{H}$ (turns to $\mathrm{M}$ )

M3 : malək Pa:kbitti ?

What's the matter, darling ? 
Another example is (3), where F asks her mother (M) to give her her (mother's) sweater but the latter prepares to leave the room without uttering a word. F explicitly points to the absence of the response in F2 /mazawbtini $\int \mathrm{i} /$ (you haven't answered me) and interprets it as a decline in F3 though the mother grants it in M1.

3. F1: hadik yanddiha mSaja

I'll take that one with me

(Silence)

F2: mazawəbtinifi

You haven't answered me

M1: diha

Take it

F3: $\quad$ Pa:ћ Slik mabyitifi

Rascal! You don't want to (give it to me)

M2: lla: ddi:ha wəllahila ddiha

No, take it. Do take it

$(\mathrm{FN})$

In fact, empirical data both in the literature and ours clearly attests that when rule I (a) is violated, selectors pursue a response using different repair devices, which too reveals the constraining characteristic of first parts of adjacency pairs, a normative feature that participants are oriented to (Schegloff \& Sacks, 1973, p. 294; see also Shegloff, 2007). One more example, which is taken from a much longer pursuit of a response to a question, is cited in (4). Upon receiving no answer to her question in N1, N prompts the experimenter, her friend, to provide one using different devices: A partial repeat in N2, an explicit mention of the missing response /qul/ (tell) as well as the repeat in N3, and one more emphatic explicit mention of the missing answer /wahḍar/ (Do speak) and finally a request to 'stop it' in N4.

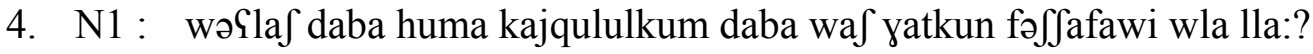

Do they tell you now whether you'll attend the oral exam or not?

$(0.83 \mathrm{sec})$

N2 : daba:?

Now?

$(1.90 \mathrm{sec})$

N3 : qu:1 daba kajqululkum $\iint a f a w i$ wa yatkun fih wla lla?

Tell me, do they tell you now whether you'll attend the oral exam or not? 
$(1.30 \mathrm{sec})$

N4 : wahḍar barak məlləbsala

Do speak! Stop it!

(REE)

\section{An extension of Sacks et al.'s model (1974)}

Given the significance of AS, which the previous examples clearly bear evidence to, this section is an attempt to provide for it within the system itself, as mentioned earlier.

Garvy \& Berninger (1981) identify sequences where AS obtains in children's conversations, and refer to them as 'notice missing response sequences', the length of which is determined by the number of attempts the current speaker makes before turn transfer occurs. Following them, we may offer the following characterization.

If a current speaker (A) uses a current speaker selects next-technique thereby putting the selected speaker (B) under the obligation to speak next, but for one reason or another, B does not take his turn leaving thus the slot in which he is expected to speak empty, then A notices the emerging silence and attempts to solicit B's response in the following slot. A sequence of the form A1 - AS - A2 thereby emerges. Turn transfer having failed after A1, the first attempt, A2, the second, serves as a repair attempt, which may or may not result in turn transfer. If the latter is the case, A may issue a third attempt A3 or a fourth A4 (An) until he obtains a response; otherwise, the conversation may discontinue.

What we have in these sequences is a pattern apparently similar to that which emerges under the operation of rule I (c). This apparent similarity did in fact tempt Coulthard (1985) into describing a notice missing response sequence where requests are responded to by silence in terms of this rule, and he describes a current speaker's pursuits of a response such as 'tags' as what Garvy \& Berninger (1981) refer to as 'post completors'. This does not sound plausible for two reasons. First, AS is a breach of rule I (a), where a current speaker selects a next one, whereas under rule 1 (c), a current speaker resumes his talk after rule 1 (b) has been passed. The two rules are clearly distinct within the model. Second, It seems inappropriate to describe response pursuits such as the marked repeat / $\mathrm{Pa}$ : $\mathrm{hm \partial d} /(\mathrm{Hey} H \mathrm{Hmed})$ in M2 in (2), the partial repeat /daba?/ (now?) in N2 in (4), the explicit mention of the noticeable absence of an answer in F2 /mazaw btinifi/ (you haven't answered me) in (3) and in H5 /katəSți șmək qul manəSțihafi/ (you're turning a deaf ear to me ; say you won't give it to me) in (1) as 'post completers', which complete the first utterance. They, instead, suggest and some even very explicitly state that the first utterance was in itself complete and that a response should have been provided before A issued his first pursuit in (A2).

Given the difference between the rules involved in cases of meaningful silence and the intra-turn pause, the outcome of the application of rule 1 (c), one may be tempted into suggesting that Sacks et al.'s (1974) rule set include one more rule in I-a new I (b) rule below (see I (a) above). The terminology is theirs. 
I - (b). If a selected speaker fails to start next turn, then the current speaker has the right to pursue a response to his utterance until he obtains one, turn-transfer being thereby effected; otherwise, the conversation may discontinue.

Accordingly, rule I (b) and I (c) in Sacks et al.'s (1974) initial rule set would be referred to as I (c) and I (d), respectively. The extended set would still consist of two main components: The current speaker selects next and the self selects techniques. Unlike in the former set, the first component would include two sub-rules: (a) and (b). This way, AS, like rule I (c) and rule I (d) silences, would be described as "the product of rules" (Schegloff, Jefferson, \& Sacks 1977, footnote, p. 382). More precisely, it would be characterized in terms of rule I (a) and the new I (b) one, and the resulting basic sequence would have the following pattern:

$$
\text { A1 (rule I (a)) - AS - A2 (rule I (b)) - B }
$$

In the absence of turn transfer upon the completion of A2, as in many of our examples, the sequence may be extended, its length being determined by the number of response pursuing attempts the current speaker makes before turn transition operates. This extension implies the revision of the second part in the rule set. Thus one could reformulate it as follows:

II. If at the initial transition relevance place of an initial turn constructional unit neither I (a) (and by implication I (b)) nor I (c) has operated, and, following the provision of I (d), the current speaker has continued, then the rule set (a) - (d) reapplies at the next transition relevance place, and recursively at each next transition relevance place, until transfer is effected.

However, this reformulation of the initial rule set, we think, is inappropriate. The initial one provides for the normal operation of the turn-taking mechanisms whereas the new set incorporates a new rule of a different status: The new 1 (b) rule provides for one case of turn-taking violations. Therefore, it would be more appropriate to include the above characterization of AS in one of the fundamental components of Sacks et al.'s (1974) model namely component (14):

Repair mechanisms exist for dealing with turn-taking errors and violations; e. g. If two parties find themselves talking at the same time one of them will stop prematurely, thus repairing the trouble (p. 701).

We can then perhaps break down component (14) into subcomponents, each addressing a different error or breach of the turn-taking rules. The characterization of AS offered above, which could be labelled 'AS repair rule', would be one of these subcomponents.

By revising the system in the way suggested above to account for a conversational event that is intrinsic to the turn-taking system and highly significant to social actors, and provided our extension has some validity, we have provided a case where Sacks et al.'s (1974) claim that "at least some of the mechanisms for turn-transfer repair are intrinsic to the very system whose trouble they repair" (Sacks et al., 1974, p. 723). AS can then be described as one of the violations "the various organizations operative in conversation are susceptible to" (Sacks et al., 1974, p. 723). It could be referred to as the "repairable" (Schegloff et al., 1977). The 
different devices the speaker produces in his pursuit of a response and preservation of conversation continuity such as repeats and partial repeats could be called, just as in the literature, as repair devices. This provision for the repair of the turn-taking system violation within the system itself is one power of the system (Levinson 1983, p. 320).

\section{Features of AS}

Another power of the turn-taking system is its ability to assign "the absence of any verbal activity to some particular participant as his turn: Such a mechanism can quite literally make something out of nothing, assigning to a silence or a pause, itself devoid of interesting properties, the property of being A's, or B's, or neither A's nor B's" (Levinson, 1983, p. 321).

Attributability is, in fact, one of the most important characteristics of AS, as explicitly indicated in the label assigned to it by Schegloff \& Sacks (1973). Unlike silence at the end of a conversation, which is the result of the fact that the transition relevance of potential utterance completion is suspended, AS is due to "its non-occurrence while still relevant" (Shegloff \& Sacks, 1973, p. 295). Scheggloff \& Sacks (1973) generally treat it as the silence of the selected party, who has violated rule I (a). In one context, however, they say: "that the silence is heard as the other's, but treated as one's own is for talk purposes a delicately interesting matter" (p. 294), and they classify it as the selector's pause. This adds to the terminological confusion discussed earlier. The "pause" in this case is the result of the violation of rule 1 (a) by the selected party whereas in the previously discussed cases, it is not a violation of any rule; it occurs before the selected party starts his turn, or after the current speaker takes up his turn in the absence of self selection by others. This treatment seems to be underlain by a characterization of the bounding talk as one turn. We do not think, however, that this type of silence can be treated as such. In fact, as the data such as example (1) above, and (5) below, which is taken from the context of bad news reporting, clearly demonstrates, participants themselves attribute it to the selected party.

5. L1 : @wifa labas @liha ?

Is Ouicha all right?

$(1.34 \mathrm{sec})$

L2 : Pah?

What?

$(0.63 \mathrm{sec})$

L3 : Pana Sa:rəfha mqallqa Slijja

I know she is angry with me

$(0.53 \mathrm{sec})$

L4 : wamalək skətti ?

Hey! Why are you silent? 
L's wife has died and a friend of his has come to report the news to him, but finds the task very difficult, which is partly shown in the delay of the report across turns. After three failed attempts at having his request for information about her responded to, L explicitly points to this silence in L4 (Hey! Why are you silent?).

The following anecdotes too very explicitly point to this silence assignment and serve to illustrate another feature of AS-namely reportability.

6. qutlu nta maSrafti Ali Sandu flu:s wla maSandu: masəwwulti: maqulti wa:lu bqa yisakət mazbar majqul

I said to him: "You didn't bother to know if Ali has any money or not; you didn't ask. You didn't say anything". You know, he just kept silent. He didn't say anything.

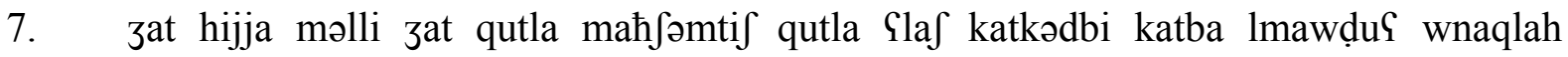
wkatquli malqiti wa:lu Srafti bqat sa:kta bqat yikatəhmar

So when she came I said to her: “Aren't you ashamed of what you did?". I said to her: "Why do you lie? You wrote the composition; you copied it down and you told me you hadn't found anything." You know, she just kept silent. She just kept blushing.

(FN)

As the anecdote in (6) makes clear, the reporter, who reproaches her co-participant, her husband, for failing to help their son 'Ali', attributes the silence emerging after her reproach to her husband, who she has selected through the first part of an accounting sequence. The same attribution is made by the reproacher in the same kind of context, where she reproaches her friend for lying to her about an assignment she has done but denies having done.

Given that participants themselves assign AS to the selected party and that A (the selector) often indicates in A2 that his prior utterance was itself complete and that B (the selected party) should have talked in the empty slot after A1, it would, we think, be more plausible to consider A1 and A2 as two turns rather than as one, a view which is in line with Garvy \& Berninger's (1981). If we so characterize these utterances, then the emerging silence would not fall within A's turn. It would not then be classified as an-intra-turn pause, and thus some of the terminological confusion discussed previously might be clarified. It could perhaps be referred to as a "missing turn silence". The selected speaker actually misses a turn as defined above, but he performs, except if he is physically or interactionally unavailable, and is usually understood to have performed an action. More specifically, our analysis of AS in different contexts in the thesis reveals that a silence after an utterance that awaits a positive/negative response such as requests for goods, actions, opinions, and suggestions tends to be interpreted as the absence of the positive act (granting for example), rather than the negative one (decline). Examples (1) above and (8) below, among others, are evidence of this, as will be shown below.

As the anecdotes in (6) and (7) further show, another characteristic of AS is reportability 
(Schegloff \& Sacks, 1973). It is often the case that participants recall this absence and report it in later conversations, which further attests to its significance to them. In such cases, the reporter usually uses expressions or verbs that indicate their interpretation of the silence. A case in point is /naxxalni/ or / țani nnuxxala/ (literally, he gave me chaff), which means 'he gave me the cold shoulder'.

One more feature of AS that is highly significant for participants' interpersonal relation is accountability. The latter is a crucial notion within conversation analysis and is based on the idea that social actors design their behavior with awareness of its accountability (Shegloff \& Sacks 1973; Schegloff, 1972, Schegloff, 2007). Upon the absence of a response in the slot following the completion of a first pair part, the current speaker usually draws particular inferences from this "official absence". Shegloff (1972) discusses this point with reference to summons-answer sequences: "A member of the society may not 'naively' choose not to answer a summons. The culture provides that a variety of 'strong inferences' can be drawn from the fact of the official absence of an answer, and any member who does not answer does so at the peril of one of these inferences being made" (p. 392)

Thus if a participant reacts to a summons by silence, the summoner is entitled by his culture to make an inference from this option, and the former knows also from his knowledge of this culture that he will be held accountable. Schegloff (1972) further observes that the summoner usually initially provides a safe account-one in terms of physical or interactional unavailability rather than in terms of improper or a-social behavior. In the absence of the former, however, a more serious account such as 'sulking', 'insulting' or 'giving the cold shoulder' generally emerges.

Example (3) above provides another instance of the accountability feature. F, in F3, explicitly states the account-the decline of the request (you don't want to) after her mother's silence, and though the latter denies this in M1, F does not believe her, as shown in F4 (Rascal! You don't want to). This seems to attest to participants' orientation to the fact that grantings of requests, one example of preferred seconds, are usually performed without delay. Briefly, preference is a structural not a psychological notion within conversation analysis. Preferred and dispreferred seconds are alternative action types or responses which represent different alignments towards the action performed in firsts and which have different structural features. A request, for instance, is either granted or rejected. Grantings tend to be unmarked and performed without delay whereas rejections are usually characterized by opposite features (for more details, see Shegloff et al., 1977, Schegloff, 2007, and Kendrick \& Torreira, 2015). One last example of the just mentioned participants' orientation is present in (8), another request sequence involving a mother $\mathrm{M}$, her son $\mathrm{A}$, and daughter $\mathrm{F}$.

8. A1: rọi Cla wlidək Pammi

$(1.82 \mathrm{sec})$

Bless your son, mum

F1 : hajha:

$\mathrm{Oh}$ ! 
$(0.46 \mathrm{sec})$

A2 : ja:k Pammi

It's not nice of you, mum

$(2.18 \mathrm{sec})$

F2 : Ju kajsৎalək rrḍa fu:f

Look, he's asking for your blessing

A3 : ja:k

It's not nice of you

$(0.76 \mathrm{sec})$

A4 : wa:xxa Slik

It's not nice of you

$(0.46 \mathrm{sec})$

A5 : makatərḍifi Glijja Pana ja wa:lu ?anaja

You don't bless me; you don't.

(TRCE)

A in A1 requests his mother for her blessing (after he shouted at her), which is highly valued in the Islamic culture, but she keeps silent even after F's appreciation of the request in F1. This he interprets as a decline in A2, A3 and A4, all of which can be classified as reproaches for the decline, and in A5, the decline is overtly expressed (you don't bless me; you don't). Kendrick and Torreira's (2015) study of preferred/dispreferred responses to invitations, offers, requests, suggestions, and proposals also provide evidence that an inter-turn silence that departs relatively largely from the normal or standard gap, one ranging between 0.7 and 0.8 sec, indicates that "rejection is imminent" (p. 287) . (It is generally agreed that the norm ranges between 0.1 and $0.3 \mathrm{sec}$; see, for example, Levinson \& Torreira, 2015 and Kendrick, 2015). It is precisely because of inferences like these, which are usually negative or a-social in cases where a positive/negative pair of options is relevant, that participants cannot be said to "naively" decide not to satisfy the selector's expectation. Thus a party who is interested in avoiding these negative inferences would respond as immediately as possible upon the completion of a first part of an adjacency pair, and would thereby reduce the silence between the selector's speech and his. In this, lies a further reason for the minimization of silence after the application of rule 1 (a).

Nonetheless, a silent participant may deny the legitimacy of a particular inference and provide a different usually safe one. It is, in fact, this safe account that participants who intend to engage in a-social acts exploit if challenged for this intention (Schegloff, 1972). Cited below is an anecdote that clearly attests to this exploitation.

9. katt $\quad$ adda Cliha mrat xaha bəzza:f məskina bqawwət $\iint \gamma u l$ tafəllaxxar bqat məlli 
katYajjəțla katəSṭiha șṣmək katqi rașa gaYma səmৎata wkatqulla wəllah masmaYtək Paxti məlli katqulla katəSṭi ș̣̦mək Pabənt ləћћram

Her sister-in-law treats her cruelly, poor girl. She gives her too much housework, so she has started to turn a deaf ear to her and pretend not to hear her. When she says "you turn a deaf ear to me, bustard!", she (her sister-in law) says "I swear I didn't hear you, sister"

Upon receiving no answer to her summons, the summoner draws the conclusion that her sister-in-law, who she treats cruelly and assigns much housework to, snubs her (you turn a deaf ear to me, bustard). The summoned party denies this account and claims a safe onephysical unavailability (I swear I didn't hear you, sister).

To conclude, after an AS, participants do not usually disregard this absence of speech. They clearly notice and assign it to the selected party, and pursue a response in their following turn (s), in which they provide different accounts depending on the nature of the adjacency pair employed and showing an orientation towards negative interpretations. They can also report it in later interactions. The regular pursuit of a relevant response is a clear reflection of the normative constraining feature of adjacency pairs mentioned above. AS in Moroccan conversation, then, is characterized by the same features as in English (Schegloff \& Sacks (1973), which is further evidence that the fundamentals of Sacks et al.'s (1974) turn-taking model work across languages and cultures.

One point worth mentioning is that most often our participants both in the experiment and the recorded conversations proceed to repair the turn-taking violation after silences longer than 1 sec. Of relevance to us in relation to this is that Jefferson (1989), in an attempt to find out of what length a tolerance interval generally is in conversation, discovers that silences ranging between 0.9 and $1.2 \mathrm{sec}$ are ones at the end of which one of the conversation partners starts to do some "resolutional activity" (p. 170).

Another interesting point is that in other cases, the participants resort to repairing the turn-taking system violation after silences ranging between 0.5 and $0.9 \mathrm{sec}$. Of particular significance is that all the utterances preceding these silences except two are marked as interrogative. Most interesting perhaps is that most of them are yes/no questions. This, on the one hand, reveals the major role of questions in reducing gaps between turns, a fact that Hanni's (1980) findings also confirm. Hanni's (1980) subjects were asked to listen to utterances taken from a dialogue and separated by an artificial $7 \mathrm{sec}$ interval and to press the button at whatever point in the utterance or the following silence they thought turn transfer might occur. The results reveal that the utterances that cause shorter latencies are, with one exception, questions. On the other hand, it seems consonant with Stiver et al.'s (2009) findings regarding the markedness value of silence after polar questions in the ten languages studied. Their results show that, on average, responses to polar questions tend to be delayed by no more than $0.5 \mathrm{sec}$, which points, according to them, to "a single shared infrastructure for language use with likely ethological foundations" (abstract). 


\section{Conclusion}

In this article, we have examined AS within conversation analysis providing examples from the Moroccan context. We have shown that though Sacks et al.'s (1974) model does not account for it, it and the conversation analysis approach in general provide interactional mechanisms, which have been used to extend the model so that it can provide for this violation of the turn-taking system within the system itself, in accordance with Schegloff \& Sacks' (1973) claim that this machinery should incorporate mechanisms for dealing with errors and violations (p. 294). We have also discussed four main features of AS-noticeability, reportability, attributability, and the socially crucial one, accountability. In addition, we have briefly pointed to what the markedness value of silence after the operation of the current speaker selects next technique could be in Moroccan conversation: $0.5 \mathrm{sec}$ seems to be a criterial cut-off point after questions especially polar questions and 1 sec after other utterances. A much larger corpus and statistical measures, however, are needed to confirm or disconfirm these results.

The study in the thesis also shows that AS is an integral part of dispreferred seconds such as declines of requests and suggestions. It also demonstrates, however, that those devices that are often characterized as dispreferreds' tokens such as hesitations and repeats are not only characteristics of dispreferred seconds but more generally of acts participants would rather not perform such as bad news reporting. To account for the use of this category of silence and its role in interpersonal relations, Taylor \& Cameron's (1987) psychological common sense definition of preference and Brown \& Levinson's (1978) theory of politeness are adopted. In brief, AS is found to function as a time buying device or an avoidance strategy which participants resort to to avoid or phrase a difficult message (rejection of requests and admission of guilt, for example), and to redress their as well as their partners' face damage. In other situations, it indicates an open rejection of co-operation. The silent participant does not have recourse to silence to delay the performance of a face threatening act or to mitigate its effect on his interlocutor. Silence is in its own right a face threatening act, a purposeful rejection of a co-participant's want to be responded to. Thus just as face preservation may be effected across a series of turns with silence being used as one means to that end, others being other verbal and non-verbal devices, face damage may operate across such a sequence with silence being the only means. Both situations are problematic, and the problem lies either in the face threatening act performed or the conflictual relation between the participants.

One important topic to further explore is the interpretation and tolerance of AS in same and cross-gender conversations, which may show whether Moroccan men and women diverge or converge along these two dimensions. Of no less importance perhaps is the study of the breach of the turn-taking norm that AS is an instance of in teenage talk, which is largely reported to be anti-normative (Elouakili, 2017; Zimmerman, 2009). Its markedness value in Moroccan conversation can also be examined within a more comprehensive and focused study using a more sophisticated method of silence measurement than the stop watch employed in the thesis. This will probably corroborate Stiver et al.'s (2009)'s claim that "there is a universal semiotics of delayed response" (conclusion, para. 1). 


\section{References}

Brown, P., \& S. C. Levinson. (1978). Universals in Language Usage: Politeness Phenomena. In E. N. Goody (Ed.), Questions and Politeness Strategies in Social Interaction (pp. 56-289). Cambridge: Cambridge University Press.

Chafe, W. (1985). Some Reasons for Hesitating. In D. Tannen \& M. Saville-Troike (Eds.), Perspectives on Silence (pp. 77-89). Norwood, New Jersey: Ablex.

Coulthard, M. (1985). An introduction to Discourse Analysis. New York: Longman.

Elouakili, S. (2017). A Linguistic Study of Borrowing in Moroccan Teenage Talk. International Research in Education, 5(1), 162-180. http://dx.doi.org/10.5296/ire.v5i1.11022

Elouakili, S. (1990). The Conversational Role of Silence in Moroccan Arabic (Unpublished MA thesis). Mohammed Ben Abdellah University. Fés. Morocco.

Gardner, R. (2010). Questions and Answer Sequences in Garrwa Talk. Australian Journal of Linguistics, 30(4), 1-37. http://dx.doi.org/10.1080/07268602.2010.518554

Gardner, R., \& Mushin, I. (2015). Expanded Transition Spaces: The Case of Garrwa. Frontiers in Psychology, 6(251). http://dx.doi.org/10.3389/fpsyg.2015.00251

Garvy, C., \& Berninger, G. (1981). Timing and Turn Taking in Children's Conversation. Discourse Processes, 7, 27-57.

Heldner, M., \& Edlund, J. (2010). Pauses, Gaps and Overlaps in Conversations. Journal of Phonetics, 38(4), 555-568.

Hanni, R. (1980). What is Planned during Speech Pauses?. In H. Giles, W. Robinson, \& P. Smith (Eds.), Language: Social Psychological Perspectives (pp. 321-325). Oxford: Pergamon Press.

Jefferson, G. (1989). Preliminary Notes on a Possible Metric which Provides for a "Standard Maximum" Silence of approximately One Second in Conversation: An Interdisciplinary Perspective. In P. R. Roger \& P. Pull (Eds.), Multilingual Matters (pp. 166-196). Philadelphia: Clevedon.

Kendrick, K. H. (2015). The Intersection of Turn-taking and Repair: The Timing of Other-initiations of Repair in Conversation. Frontiers in Psychology, 6(250).

Kendrick, K. H., \& Torreira, F. (2015): The Timing and Construction of Preference: A Quantitative Study, Discourse Processes, 52(4). 255-289. http://dx.doi.org/10.1080/0163853X.2014.955997.

Kenny, C. (2011). The Power of Silence: Silent Communication in Daily Life. London: Karnac Books.

Levinson, S. C. (1983). Pragmatics. Cambridge: Cambridge University Press.

Levinson, S. C., \& F, Torreira (2015). Timing in Turn-taking and its Implications for 
Processing Models of Language. Fontiers in Psychology, 6(731). http://dx.doi.org/10.3389/fpsyg.2015.00731

Mushin, I., \& Gardner, R. (2009). Silence is Talk: Conversational Silence in Australian Aboriginal Talk-in-interaction. Journal of Pragmatics, 41(10), 1-46. http://dx.doi.org/10.1016/j.pragma.2008.11.004.

Gardner, R., \& Mushin, I. (2015). Expanded Transition Spaces: The Case of Garrwa. Fontiers in Psychology, 6(251). http://dx.doi.org/10.3389/fpsyg.2015.00251

Owen, M. (1981). Conversational Units and the Use of 'Well ...'. In P. Werth (Ed.), Conversation and Discourse (pp. 99-116). London: Groom Helm.

Power, R., \& Martello, M. (1986). Some Criticisms of Sacks, Shegloff \& Jeffersson on Turn-Taking. Semiotica, 58, 29-40.

Sacks, H., Schegloff, E., \& Gefferson, G. (1974). A Simplest Systematics for the Organization of Turn Taking for Conversation. Language, 50. 696-735.

Schegloff, E. (1972). Sequencing in Conversational Opening in Communication. In J. Laver \& S. Hucheson (Eds.), Face-to Face Interaction (pp. 374-405). Great Britain: Penguin Books.

Schegloff, E. (1982). Discourse as an Interactional Achievement: Some Uses of "Uh huh" and Other Things that Come between Sentences. In D. Tannen (Ed.), Analysing Discourse, Texts, and Talk (pp. 71-93). Washington: Georgetown University Press.

Schegloff, E. (2007). Sequence Organization in Interaction. A Primer in Conversation Analysis. Cambridge University Press. Retrieved from www.cambridge.org

Schegloff, E. (2010). Commentary on Stivers and Rossano: "Mobilizing Response". Research on Language and Social Interaction, 43(1), 38-48. http://dx.doi.org/10.1080/08351810903471282.

Schegloff, E. (2015). Conversational Interaction. The Embodiment of Human Sociality. In T. Tannen, E. H., Hamilton, \& D. Schiffrin (Eds.), The Handbook of Discourse Analysis (pp. 346-366).

Schegloff, E., Jefferson, G., \& Sacks, H. (1977). The Preference for Self-correction in the Organization of Repair in Conversation. Language, 53(2), 367-382.

Schegloff, E., \& Sacks, H. (1973). Opening up Closings. Semiotica, 8. 289 -327.

Shrader-kniffki, M. (2007). Silence and Politeness in Spanish and Zapotec Interactions (Oaxaca, Mexico). In M. E. Plencia \& C. Garcia (Eds.), Research on Politeness in the Spanish Speaking World (pp. 305-332). Mahwah: Lawrence Erlbaum.

Stivers, T. et al. (2009). Universals and Cultural Variation in Turn-Taking in Conversation. http://dx.doi.org/10.1073/pnas.0903616106

Taylor, T., \& Cameron, D. (1987). Analysing Conversation: Rules and Units in the Structure 
of Talk. Language and Communication Library. 9. Oxford: Pergamon Press.

Zimmerman, K. (2009). A Theoretical Outline for Comparative Research on Youth Language with an Outline of Diatopic-contrast Research within the Hispanic World. In A. B. Stenström \& A. M. Jørgensen (Eds.), Youngspeak in a Multilingual Perspective. (pp. 137-160).

\section{Appendix}

Key to the transcription symbols

\section{Vowels}

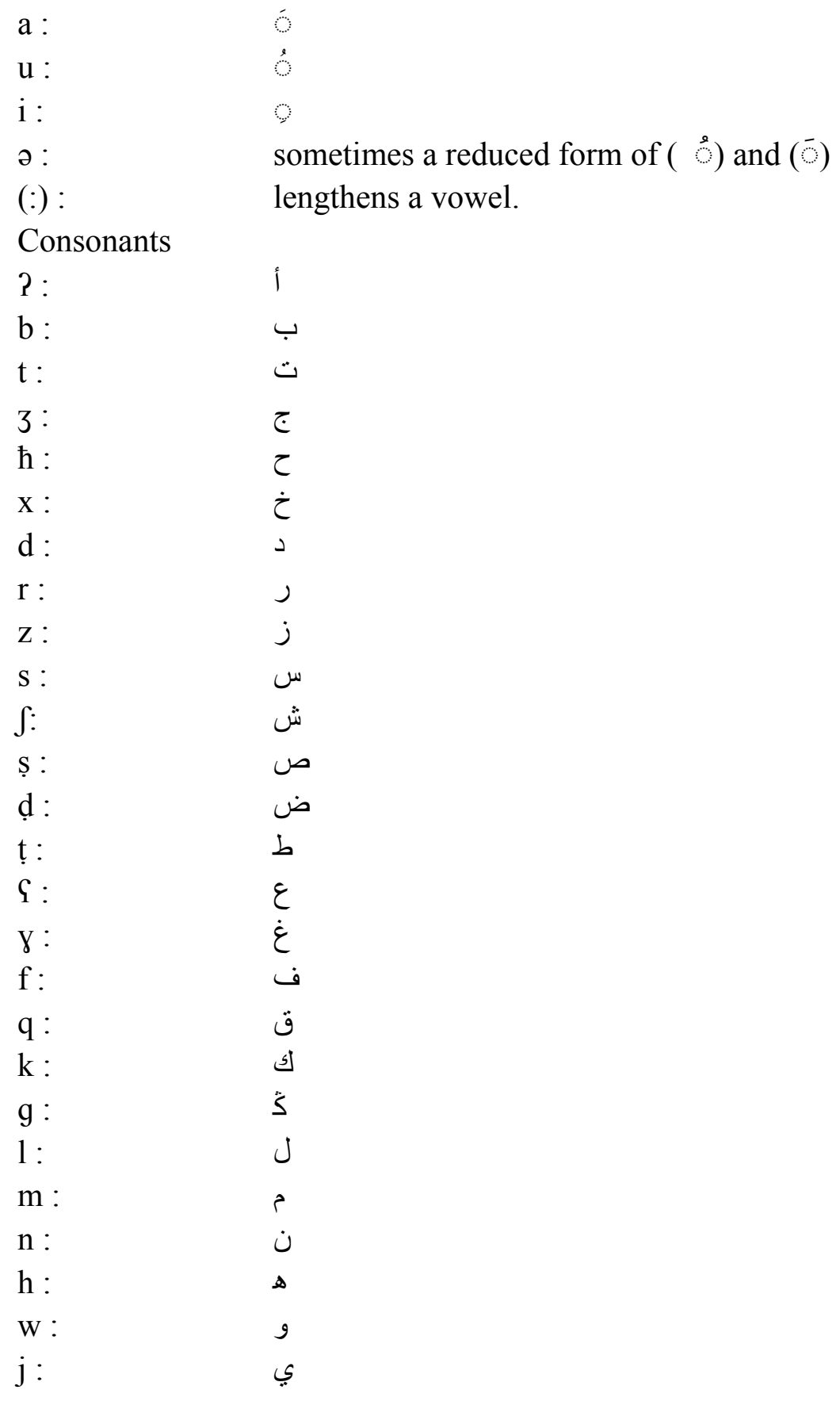


Key to the abbreviations for the data sources

$(\mathrm{FN})$ :

Field note

(NREE):

Non-recorded excerpt from the experiment

(REE):

Recorded excerpt from the experiment

(ESO):

Excerpt from a soap opera

(TRCE):

Tape recorded conversational excerpt

\section{Copyright Disclaimer}

Copyright reserved by the authors.

This article is an open-access article distributed under the terms and conditions of the Creative Commons Attribution license (http://creativecommons.org/licenses/by/3.0/). 